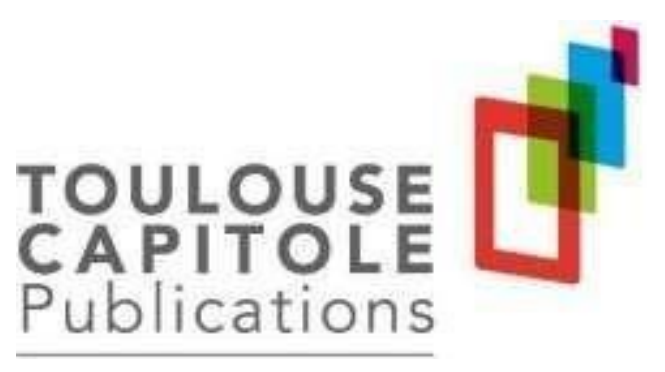

«Toulouse Capitole Publications » est l'archive institutionnelle de l'Université Toulouse 1 Capitole.

De l'urgence à définir l'urgence en kinésithérapie

\author{
JEAN SEVERIN \\ ROCTON ROLAND
}

Référence de publication : Jean, Séverin, Rocton, Roland, «De l'urgence à définir l'urgence en kinésithérapie », Kinésithérapie, la Revue, 2017, 17 (192). p. 51-56. 


\section{De l'urgence à définir l'urgence en kinésithérapie}

\section{Résumé}

Lorsqu'ils interviennent dans un but thérapeutique, les kinésithérapeutes exercent leur art sur prescription médicale. La nouvelle rédaction de l'article L.4321-1 du Code de la santé publique, issu de la loi du 26 janvier 2016, introduit une circonstance dérogatoire à cette règle générale : en cas d'urgence et en l'absence d'un médecin, les kinésithérapeutes sont habilités à mettre en œuvre sans prescription médicale les premiers actes de soin en Masso-Kinésithérapie. Cette dérogation est nécessairement d'interprétation stricte et ne permet l'accès direct qu'en présence de ses deux conditions cumulatives : l'absence d'un médecin et une situation d'urgence. L'urgence, n'étant pas définie juridiquement, renvoie à la notion de « préjudice dans le retard ». Dès lors, il appartiendra au kinésithérapeute d'apprécier au cas par cas si son intervention se situe dans ce cadre. La question à laquelle celui-ci devra répondre se résume à : « y a-t-il un risque à différer les actes de kinésithérapie ? »Dans l'affirmative, la nécessité d'une intervention sans délai (intérêt supérieur) permettra de déroger à l'exigence d'une prescription médicale (intérêt inférieur). Le kinésithérapeute pourra alors pratiquer les premiers soins de kinésithérapie nécessaires pour répondre à la situation d'urgence. En cas de contentieux, il appartiendra au juge du fond de déterminer, au besoin à l'aide d'une expertise technique de kinésithérapie, si, compte tenu des circonstances de l'espèce, le masseur-kinésithérapeute est bien intervenu dans le cadre défini par l'article L.4321-1 du code précité. La modification de l'article commenté formalise, par ailleurs, l'existence d'une urgence spécifique, l'urgence médicale de Masso- Kinésithérapie, distincte de l'urgence médicale générique et à laquelle seul le masseurkinésithérapeute peut répondre en raison de ses compétences spécifiques. Néanmoins, de nombreuses questions découlent de ces modifications (information du patient, consentement éclairé, prise en charge financière par les organismes sociaux, etc.), qui demanderaient une étude dépassant le cadre de cet article, qui ne peut être qu'un commencement.

Y-a-t-il urgence à définir l'urgence ? Assurément depuis que la loi du 26 janvier 2016 de modernisation de notre système de santé1 a donné lieu à la création de l'article L.4321-1 in fine du Code de la santé publique : «En cas d'urgence et en l'absence d'un médecin, le masseur-kinésithérapeute est habilité à accomplir les premiers actes de soins nécessaires en Masso-Kinésithérapie. Un compte rendu des actes accomplis dans ces conditions est remis au médecin dès son intervention ». L'insertion d'une telle disposition dans le Code de la santé publique offre des perspectives nouvelles pour la profession de masseur-kinésithérapeute dans la mesure où, selon l'interprétation retenue, il sera possible ou non pour celle-ci de pratiquer des actes de Masso-Kinésithérapie en dehors de toute prescription médicale. En effet, l'article L.4321-1 in fine du Code de la santé publique invite à se demander, comme cela semble être le cas, si un masseur-kinésithérapeute, confronté à une situation d'urgence, peut accomplir des actes de soins nécessaires en Masso-Kinésithérapie hors prescription médicale ? En réalité, ce n'est pas tant à cette question qu'il convient de répondre dans la mesure où la loi y répond favorablement et expressément, mais de déterminer dans quel- les conditions le masseur-kinésithérapeute est habilité à accomplir les premiers actes de soins nécessaires en Masso-Kinésithérapie face à une situation d'urgence ?

De toute évidence, l'enjeu principal réside dans la définition de l'« urgence » (I) qui apparaît, à côté de l'exigence de « l'absence d'un médecin », comme la condition préalable à remplir pour autoriser 
l'intervention - hors prescription médicale - du masseur-kinésithérapeute dès lors que son intervention est de nature thérapeutique2. Ce n'est qu'à cette condition préalable qu'il sera possible d'envisager les effets de la définition juridique de l'urgence (II).

\section{I- LA DÉFINITION JURIDIQUE DE L'URGENCE}

L'un des plus éminents juristes du XXe siècle, Philippe Jestaz, dans sa thèse de doctorat relative à l'urgence, écrivait déjà que « définir l'urgence d'une façon rigoureuse est une entreprise vouée à l'échec. La plupart des auteurs qui s'y sont essayé n'ont abouti qu'à des formules imprécises qui gravitent autour de l'idée d'un préjudice dans le retard » [1, no 9]. Cette affirmation n'a nullement perdu de perspicacité puisque tant la doc- trine juridique que la jurisprudence n'ont réussi, pour l'heure, à définir précisément l'urgence.

L'insaisissabilité de la notion tient à son défaut de stabilité, d'uniformité. En effet, il n'y a pas une urgence, mais des urgences : urgence à ester en justice afin d'éviter la prescription de l'action, urgence à gérer les affaires d'autrui afin que ce dernier ne voit sa situation s'aggraver ou encore, par exemple, urgence médicale. Plus encore, la notion d'urgence échappe au contrôle de la Cour de cassation parce que l'idée d'urgence est davantage ressentie que définie ; parce que les faits qui la constituent excluent toute systématisation. Autrement dit, le contrôle de l'urgence est dévolu aux juridictions du fond puisque l'urgence est une notion de pur fait. Cela étant, « le droit, qui est l'art d'organiser les rapports sociaux, ne peut ignorer qu'elle a d'innombrables applications pratiques » [1, no 1]. En effet, l'urgence devient une notion juridique lorsqu'elle est saisie par une règle de droit, laquelle, en la matière, est bien souvent une cause générale d'abréviation des délais et de simplification des formes à l'image ici de l'absence de prescription médicale qui est pourtant la règle, le principe lorsque la masseur-kinésithérapeute intervient à des fins thérapeutiques.

Peut-on alors imaginer une définition juridique de l'urgence?

La doctrine juridique s'entend au moins sur un point : l'urgence renvoie à l'idée de préjudice dans le retard. Cette expression a au moins le mérite de mettre en évidence deux éléments fondamentaux dans la constitution de l'urgence. D'une part, le préjudice qui peut être plus ou moins grave ; d'autre part, l'expression «dans le retard » invite à interroger l'urgence du point de vue de sa temporalité. Ainsi, il conviendra, pour que le professionnel de santé - le masseur-kinésithérapeute - juge de l'urgence en articulant ces deux expressions pour s'autoriser à intervenir hors prescription médicale. Il ressort de l'expression « préjudice dans le retard » que l'urgence ne se définit non par sa cause, mais davantage par son but. Par conséquent, il ne semble guère utile de rechercher une définition juridique de l'urgence, tout comme il semble inopportun d'aller rechercher des synonymes de l'urgence3 dont le trait commun réside d'ailleurs toujours dans l'idée de préjudice dans le retard. En définitive, l'urgence en droit doit être définie fonctionnellement à travers l'idée de préjudice dans le retard.

Cette définition retenue invite à retenir deux éléments constitutifs de l'urgence : d'une part, l'urgence suppose une menace à écarter qui suppose l'action du masseur-kinésithérapeute (A) ; d'autre part, cette action s'oppose à un obstacle de droit : la prescription médicale (B). 


\section{A - La menace à écarter ou l'action du masseur- kinésithérapeute}

«L'urgence est constituée lorsque l'attente implique la possibilité d'un préjudice et, au mieux, le maintien de l'état de choses antérieur » [1, no 273].

Ce point est fondamental parce qu'il exprime l'idée selon laquelle il n'y a pas une urgence, mais des urgences selon l'importance du préjudice, réalisé ou à venir assurément, qui implique l'action immédiate du masseur-kinésithérapeute, soit pour éviter que le préjudice réalisé ne s'aggrave, soit que le préjudice ne se réalise. L'urgence sera d'autant plus grande que la réalisation de la menace, c'est-à-dire du préjudice ou de l'aggravation du préjudice à éviter, sera plus probable et plus proche. Or, la détermination d'un seuil objectif sera nécessairement arbitraire de sorte que l'appréciation de la menace à écarter relève du diagnostic réalisé, avec plus ou moins de temps selon la temporalité de l'urgence, par le masseurkinésithérapeute. Toutefois, il ne faudrait pas croire que l'urgence échappe à toute objectivité, " elle consiste toujours en la menace (parfois ténue) d'un dommage lié à l'écoule- ment du temps » [1, no 313]. Il ressort de l'analyse que la menace à écarter conduit, parce qu'il n'y a pas une urgence mais des urgences, à envisager l'objet auquel elle s'applique pour tenter d'en délimiter son périmètre. Pour ce faire, il faut revenir à l'article L.4321-1 in fine du Code de la santé publique commenté. À cet égard, l'exigence de l'absence de médecin, pour que le masseur- kinésithérapeute puisse pratiquer les premiers actes de soins nécessaires en Masso-Kinésithérapie, invite à considérer le domaine de l'urgence, c'est-à-dire son objet. Or, le même article dispose que « la définition des actes professionnels de Masso-Kinésithérapie, dont les actes médicaux prescrits par un médecin, est précisée par un décret en Conseil d'État (.. .) ». Dès lors, il faut en déduire que si tous les actes professionnels de Masso-Kinési- thérapie ne sont pas des actes médicaux, ceux prescrits par le médecin le sont du seul fait de cette prescription.

Or, l'article L.4321-1 in fine du code précité envisage l'urgence en l'absence de médecin, ce qui laisse sous-entendre que le masseur-kinésithérapeute peut réaliser, au nom de l'urgence, des actes médicaux relevant de la masso-kinés- thérapie sauf à considérer que les actes de Masso-Kinési- thérapie ne sont des actes médicaux qu'en raison de la formalité de principe qui n'est autre que la prescription médicale dès lors qu'il s'agit d'actes à destination thérapeutique. Cela étant, dans la mesure où l'interdiction ne vise que les actes soumis à prescription médicale réalisés à des fins thérapeutiques, il y a tout lieu de penser que les actes de Masso-Kinésithérapie doivent recevoir la qualification d'actes médicaux sans quoi on comprendrait mal qu'ils exigent ladite prescription médicale.

Quoi qu'il en soit, dès lors que l'intervention, au nom de l'urgence, poursuit une fin thérapeutique, et peu important que l'on parle d'actes médicaux ou de « dispositifs médicaux »4, l'interprétation de ces textes implique de traiter de l'urgence non pas de manière indéterminée mais de façon déterminée, c'est-à-dire eu égard à l'urgence médicale. En effet, c'est ce que laisse penser l'exigence de l'absence de médecin comme condition préalable à l'action du masseur- kinésithérapeute.

L'urgence dont il s'agit serait alors une urgence médicale.

«L'urgence médicale traduit cet instant soudain où la vie peut basculer vers la mort et la bonne santé vers l'infirmité » [2, no 1]. Plus précisément, " l'urgence médicale représente la qualité d'une situation qui résulte de la réalisation soudaine d'un événement susceptible d'entraîner, à défaut d'une intervention secourable rapide, un préjudice grave, voire irréparable à la vie ou à la santé d'une personne » [2, no 35]. L'urgence médicale peut se définir graduellement selon deux schémas qui renvoient en réalité à l'expression « préjudice dans le retard »: 
D'une part, « elle se décline en fonction du degré de gravité d'un état de santé. Le Docteur Pelloux distingue dans ce cadre l'urgence vitale ou détresse vitale, pour laquelle un pronostic vital est engagé, et l'urgence potentielle, susceptible de s'aggraver s'il n'y est pas porté remède rapide- ment » [2, no 8]. Ainsi par exemple, il suffit de songer, pour l'urgence vitale, à un enfant souffrant de bronchiolite, extrê- mement encombré, cyanosé. Dans cette situation, pour éviter qu'un risque vital ne se réalise, le masseur-kinésithérapeute doit mettre en œuvre des techniques de dés- encombrement. Quant à l'urgence potentielle, imaginons une personne souffrant d'une entorse de la cheville produisant une douleur insupportable ou un œdème extrême- ment important avec, dans le cas de la cheville, l'impossibilité de poser le pied par terre. Dans ce cas, en respectant bien évidemment les critères d'exclusion, l'intervention, la plus rapide possible, par le masseur-kinésithérapeute sera nécessaire ;

D'autre part, « l'urgence médicale se décline en fonction du temps imparti pour y répondre. On y distingue l'urgence absolue, qui commande un traitement médical dans un délai de six heures, l'urgence relative, dont le délai de réponse s'élève à douze heures et enfin, l'urgence différée, qui nécessite un traitement dans les 24 heures » [2]. Il semble aux signataires que ces classifications de l'urgence médicale pourraient être transposées à l'urgence médicale de Masso-Kinésithérapie, ne serait-ce afin que les masseurskinésithérapeutes envisagent concrètement les situations dans lesquelles leurs interventions ne seraient pas soumises à prescription médicale. Or, une fois l'urgence médicale de Masso-Kinésithérapie identifiée, encore faut- il y répondre par une action immédiate qui, au regard de l'article L.4321-1 in fine du Code de la santé publique se heurte à un obstacle de droit ; ce dernier étant le second élément constitutif de l'urgence.

\section{B - L'obstacle de droit ou la prescription médicale}

L'obstacle de droit est, quant à lui, facilement identifiable puisqu'il s'agit de l'exigence d'une prescription médicale dès lors que le masseur-kinésithérapeute est confrontée à l'urgence et s'apprête à intervenir à des fins thérapeutiques. En effet, l'article L.4321-1 du Code de la santé publique est très clair : " lorsqu'il agit dans un but thérapeutique, le masseur-kinésithérapeute pratique son art sur prescription médicale (.. .) ». Or, l'urgence médicale de Masso- Kinésithérapie va promouvoir un acte - l'intervention du masseurkinésithérapeute qui va réaliser les premiers actes soins nécessaires en Masso-Kinésithérapie - contraire à l'application de la règle positive, c'est-à-dire l'exigence d'une prescrip- tion déduite de la lecture a contrario de l'article L.4321-1 in fine du code précité imposant l'absence de médecin. Autrement dit, « l'urgence n'a d'effet, et par conséquent d'existence juridique, que si l'action du particulier [ici le masseurkinésithérapeute] se heurte à l'obstacle du droit [ici l'exigence de prescription médicale] et uniquement dans la mesure où l'obstacle peut être levé [ici l'article L.4321-1 in fine du Code de la santé publique] » $[1$, no 3$]$.

En réalité, la levée de l'exigence de prescription médicale - l'obstacle de droit - ne tient qu'à la confrontation des intérêts en présence. D'un côté, l'exigence d'une prescription médicale ; de l'autre, une menace à écarter, c'est-à- dire une urgence médicale. Pour savoir si l'obstacle juridique peut être franchi, il faut voir si l'intérêt - le préjudice dans le retard - qui commande les mesures de l'urgence est supé- rieur ou non à l'obstacle juridique. Ainsi, par exemple, en matière d'urgence médicale5, il est évident que le risque de perdre la vie tendà affirmer que le consentement du malade, pourtant requis, doit s'effacer pour la simple raison que notre société fait de la vie humaine un intérêt bien supérieur au consentement du malade lorsqu'il ne peut être obtenu6. Or, procédant du même raisonnement, dès lors que l'urgence médicale de Masso-Kinésithérapie (intérêt supérieur) est constatée par le masseur-kinésithérapeute, ce 
der- nier est en droit de se passer d'une prescription médicale (intérêt inférieur), qui ne peut être obtenue en raison de l'absence d'un médecin, pour agir parce que l'urgence le commande.

En définitive, on peut donner à l'urgence une valeur opérante puisqu'il y aura urgence « lorsque la protection rapide du droit ou de l'intérêt menacé est utile et parce qu'elle s'effectue qu'aux dépens d'un intérêt ou d'un droit de valeur moindre » [1, no 244].

\section{II- LES EFFETS DE LA DÉFINITION JURIDIQUE DE L'URGENCE}

L'effet principal de l'urgence, ici de l'urgence médicale de Masso-Kinésithérapie, consiste à déroger une règle de droit7, ici l'exigence d'une prescription médicale.

Rappelons à ce propos que depuis l'arrêté du 22 février 2000- modifiant l'arrêté du 6 janvier 1962, cette prescription n'est plus qualitative ni quantitative. Elle ne contraint donc pas le kinési- thérapeute quant aux actes techniques à mettre en œuvre et constitue une simple indication de recours à la kinésithérapie. Si l'urgence est en soi une question de pur fait, laissée à l'appréciation de masseur-kinésithérapeute, voire des juges du fond en cas de contentieux, « elle ne devient notion de droit, - paradoxalement, qu'en dispensant d'appliquer la règle » [1, no 328$]$.

Or, la question qui se pose est de savoir pourquoi l'urgence joue un rôle dérogatoire à la prescription médicale ? La réponse est évidente : « la règle initiale [l'exigence de prescription médicale], en cas d'urgence, va manquer son but si on ne lui substitue pas une règle dérogatoire » [1, no 352]. Cela étant, aucun juriste ne contestera que l'exception, retenue par l'effet dérogatoire d'une règle de droit, est nécessairement d'interprétation stricte8. Ainsi, il n'est pas suffisant d'affirmer le caractère dérogatoire de l'article L.4321-1 in fine du Code de la santé publique, encore faut-il se livrer à l'interprétation de son effet dérogatoire à travers l'exigence de l'urgence médicale de Masso-Kinésithérapie (A) qui doit conduire à apprécier strictement l'expression « les premiers actes de soins nécessaires en Masso-Kinésithérapie » (B).

\section{A - De l'urgence médicale de Masso- Kinésithérapie}

Les soussignés ne peuvent qu'opposer l'article L.4321-1 in fine du Code de la santé publique à l'article R.4321-10 du même code, lequel dispose qu'en « en cas d'urgence et en l'absence d'un médecin, le masseur-kinésithérapeute est habilité à accomplir les gestes de secours nécessaires jusqu'à l'intervention d'un médecin ». À bien y regarder, les deux textes conditionnent le jeu de l'effet dérogatoire aux mêmes exigences : urgence et absence d'un médecin.

En revanche, les textes diffèrent à deux égards. D'abord, l'article R.4321-10 du code précité offre la possibilité au masseur-kinésithérapeute d'accomplir les gestes de secours nécessaires là où l'article commenté vise seulement «les premiers actes de soins nécessaires en Masso-Kinésithérapie ». Si les gestes de secours prodigués au titre de l'urgence peuvent être des actes de soins de masso-kinésithérapie, tous les gestes de secours ne sont pas des actes de soins de Masso-Kinésithérapie.

En d'autres termes, il est certain que l'article L.4321-1 in fine du Code de la santé publique limite l'effet dérogatoire à la seule urgence médicale de Masso-Kinésithérapie. 
La référence aux seuls soins de Masso-Kinésithérapie permet certes de délimiter les actes attendus du masseur-kinésithérapeute, mais la rédaction est quelque peu étonnante. En effet, le Code de la santé publique organise notamment la répartition des compétences des professionnels de santé ; celles du masseur-kinésithérapeute dans une section 1 consacrée aux « actes professionnels » 9 et celles du médecin. Or, le médecin, à l'exception peut-être du médecin en rééducation fonctionnelle, n'est pas un masseurkinésithérapeute qui est le seul habilité à effectuer les actes de Masso-Kinésithérapie. Autrement dit, le masseur-kinésithérapeute, comme le médecin, dispose d'une compétence spécifique dont il a le monopole. Aussi, on voit mal comment le médecin pourrait répondre à une urgence de Masso-Kinésithérapie alors même qu'il s'agit d'une compétence dont il ne dispose pas. L'exigence alors d'une prescription médicale pour les actes de soins de Masso-Kinésithérapie appert déjà comme paradoxale sans qu'il soit utile de maintenir cette obligation en cas d'urgence médicale de Masso-Kinésithérapie. C'est d'ail- leurs sans doute pour cela que les deux textes précités diffèrent à un autre égard : l'intervention du médecin. En effet, tandis que l'article R.4321-10 du Code de la santé publique précise que les gestes de secours doivent être administrés jusqu'à l'intervention d'un médecin, l'article L.4321-1 in fine du même code n'y procède pas ou, tout du moins, ne l'évoque que dans un second temps : lorsqu'il s'agit de remettre au médecin un compte rendu des actes qui ont été accomplis en situation d'urgence.

Par conséquent, on peut en déduire qu'en raison de la compétence spécifique du masseur-kinésithérapeute, lui seul est apte à intervenir en cas, non pas d'une urgence médicale quelconque, mais en cas d'urgence médicale de Masso-Kinésithérapie. Cette précision est importante puis- qu'elle devrait avoir des conséquences dans l'appréciation des éléments de l'urgence par les juges du fond dans l'hypothèse d'un contentieux.

\section{B - L'interprétation stricte de l'expression : «les premiers actes de soins nécessaires en Masso-} Kinésithérapie »

L'effet dérogatoire de l'article L.4321-1 in fine du Code de la santé publique doit être apprécié strictement. Aussi, il est indispensable de dire quelques mots au sujet de l'expression « les premiers actes de soins nécessaires en Masso-Kinésithérapie ». Au-delà du fait qu'il paraît au signataire acquis qu'il ne s'agit que des actes de soins de Masso-Kinésithérapie, c'est surtout les « actes de soins nécessaires » qui interrogent.

En effet, «l'urgence ne justifie que les mesures strictement nécessaires pour écarter le préjudice envisagé $»[1$, no 334].

Plus encore, « toute situation qui implique un risque de dom- mage sans aucune chance de gain corrélatif impose comme nécessaire le but qui consiste à éviter le dommage. Ce but étant fixé, l'acte qui le réalisera sera lui-même nécessaire ou opportun par rapport à cette réalisation, quoique là encore il faille parler d'opportunité s'il existe un choix entre plusieurs moyens » [1, no 314].

Deux observations s'imposent :

D'une part, par actes de soins nécessaires en Masso-Kinési- thérapie, il faut sans doute comprendre ceux qui sont seule- ment aptes à répondre à l'urgence de Masso-Kinésithérapie. C'est d'ailleurs sans doute pourquoi, le législateur vise exclusivement « les premiers [nous soulignons] actes de soins »; 
D'autre part, par actes de soins nécessaires en Masso- Kinésithérapie, il faut aussi comprendre par là qu'il appartiendra au masseur-kinésithérapeute de choisir le dispositif de Masso-Kinésithérapie le plus adapté à la situation d'urgence lorsque plusieurs dispositifs, relevant de son champ de compétence, lui sont offerts.

\section{Libres propos conclusifs}

En définitive, l'article L.4321-1 in fine du Code de la santé publique a pour mérite de mettre en évidence qu'il existe des urgences médicales spécifiques au titre desquelles figure l'urgence médicale de MassoKinésithérapie. Bien qu'encadrée, l'urgence médicale de Masso-Kinésithérapie exige une réponse adéquate que seul le masseur-kinésithérapeute semble pouvoir apporter pour la simple raison que lui seul dispose de compétences de diagnostic pour ce type d'urgence et de moyens, c'est-à-dire d'actes de soins en Masso-Kinési- thérapie, pour y faire face.

Si le législateur n'a pas voulu ériger en principe le recours à un masseur-kinésithérapeute en l'absence de prescription médicale, il faut bien considérer que ce dernier dispose d'une compétence spécifique dont ne dispose pas, par principe, le médecin. Demande-t-on à un plombier de faire de l'électricité, à un électricien de faire de la plomberie quand bien même les deux interviendraient sur le même chantier de construction ? Assurément non!

Aussi, si on comprend mal pourquoi la prescription médicale est la règle en matière d'actes de soins de Masso-Kinésithérapie, lorsque ces derniers poursuivent une fin thérapeutique, dans la mesure où ils relèvent de l'appréciation du masseur-kinésithérapeute en tant que professionnel de santé ; on comprend mal davantage pourquoi la prescription médicale serait difficilement écartée en présence d'une urgence médicale de Masso- Kinésithérapie.

Cette règle dérogatoire prévue à l'article L.4321-1 du Code de la santé publique n'est pas pour autant un « chèque en blanc » laissé au masseur- kinésithérapeute.

Ce dernier devra, avant d'intervenir et selon l'urgence médicale de Masso-Kinésithérapie, établir un « diagnostic avec le plus grand soin, en s'aidant dans toute la mesure du possible des méthodes scientifiques les mieux adaptées »10. À la suite du diagnostic, il devra agir, mais pas agir en dehors de toute réglementation. En effet, il devra « dans les limites fixées par la loi, (.. .) libre[ment choisir] (.. .) [les] actes (.. .) qu'il estime les plus appropriés en la circonstance »11, c'est-à-dire eu égard à l'urgence médicale de Masso-Kinésithérapie. Plus encore, il devra, pour déterminer son acte de soin de Masso-Kinésithérapie procéder à un bilan " coûts-avantages » afin d'éviter de faire courir, à la personne en situation d'urgence médicale de Masso-Kinésithérapie, un risque injustifié12. Ainsi, quand bien même il s'agirait, par exemple, d'une véritable urgence médicale de Masso-Kinésithérapie, il se peut parfaitement que le masseur-kinésithérapeute se refuse à agir considérant que l'acte de Masso-Kinésithérapie projeté serait insuffisant à préserver l'urgence vitale de la personne menacée.

Et puis, pour celles et ceux qui redouteraient d'avoir, à partir de l'article L.4321-1 du Code de la santé public, ouvert la «boîte de Pandore », il faut avoir conscience qu'en cas de contentieux, le juge est tout autant un professionnel que le masseur- kinésithérapeute. Le juge cherchera, à partir d'expertises judiciaires réalisées par des professionnels compétents - les masseurs-kinésithérapeutes, si le masseurkinésithérapeute a commis une faute eu égard à ce que l'on pouvait attendre de lui et compte tenu des circonstances de l'urgence médicale de Masso-Kinésithérapie. 
Au-delà de cet aspect qui mériterait que l'on s'y attarde à l'avenir, de nombreuses questions devront être examinées : Quid du consentement de la victime en situation d'urgence médicale de Masso-Kinésithérapie ? Faut-il distinguer selon que la victime peut ou pas consentir aux premiers soins nécessaires en MassoKinésithérapie ? Que faire lorsque la victime s'oppose à l'exercice de ces premiers soins ? Quid de l'information due à la victime en situation d'urgence médicale de Masso-Kinésithérapie ? Quid de la responsabilité - civile, administrative, pénale ou disciplinaire - du masseur-kinési- thérapeute ayant agi dans le cadre de l'urgence médicale de Masso-Kinésithérapie ?13 Quid encore de la prise en charge financière des premiers soins nécessaires en Masso-Kinési- thérapie hors prescription ?

Finalement, cette étude ne peut être qu'un commencement qu'il conviendrait de poursuivre plus largement. Cela étant, tout comme en matière de dommage corporel, l'expertise du masseur-kinésithérapeute est spécifique et complète celle du médecin pour la simple raison qu'il dispose de compétences spécifiques. Aussi, ne peut-on qu'espérer que ces compétences soient mises à profit et au service de la personne ; laquelle bénéficie, faut-il le rappeler, du droit fondamental à la protection de la santé14, y compris en cas d'urgence. 


\section{Notes de bas de page}

1Loi no 2016-41.

2 La précision est importante puisque l'exigence d'une prescription médicale ne s'impose que « lorsqu'il agit dans un but thérapeutique » (Article L.4321-1 du Code de la santé publique).

3 «Les termes suivants ne paraissent pas devoir être distingués de l'urgence : péril, péril en la demeure, célérité, extrême urgence, péril en la moindre demeure. Tous impliquent l'idée de préjudice dans le retard, les gradations que l'on prétend établir restant insaisissables » (Philippe Jestaz, ibid., no 308).

4 Article L.4321-1 du Code de la santé publique.

5 «L'urgence médicale constitue certainement l'urgence suprême, non pas tant pour la rapidité d'intervention qu'elle réclame souvent que pour le risque couru qui est la perte de la vie (.. .) » (Philippe Jestaz, ibid., no 275).

6 C'est ce qu'exprime l'article 16-3 du Code civil lorsqu'il dispose qu'« il ne peut être porté atteinte à l'intégrité du corps humain qu'en cas de nécessité médicale pour la personne (.. .)».

7 En ce sens, v. Philippe Jestaz, ibid., no 2 ou encore Julie Moreau, op. cit., no 30.

8 «Le principe est que l'exception est d'interprétation étroite a essentiellement pour objet de prohiber une extension analogique de la règle dérogatoire » (Philippe Jestaz, ibid., no 335).

9 Article R.4321-1 à R.4321-13 du Code de la santé publique.

10 Article R.4321-81 du Code de la santé publique.

11 Article R.4321-59 du Code de la santé publique.

12 En ce sens, l'article R.4321-88 du Code de la santé publique : « Le masseur- kinésithérapeute s'interdit, dans les actes qu'il pratique comme dans les dispositifs médicaux qu'il prescrit, de faire courir au patient un risque injustifié ».

13 La responsabilité du masseur-kinésithérapeute devra être notamment appréciée eu égard à l'article R.4321-113 du Code de la santé publique, lequel dispose que « tout masseur-kinésithérapeute est habilité à dispenser l'ensemble des actes réglementés. Mais il ne doit pas, sauf circonstances exceptionnelles, entreprendre ou poursuivre des soins, ni prescrire dans des domaines qui dépassent ses compétences, ses connaissances, son expérience et les moyens dont il dispose ».

14 Article L.1110-1 du Code de la santé publique. 